\title{
An in vivo functional genetic screen reveals a role for the $T R K-T 3$ oncogene in tumor progression
}

\author{
Michael J Edel ${ }^{1}$, Avi Shvarts ${ }^{1,3}$, Jan Paul Medema ${ }^{2}$ and René Bernards*,1 \\ ${ }^{1}$ Division of Molecular Carcinogenesis, Center for Biomedical Genetics, The Netherlands Cancer Institute, Plesmanlaan 121, 1066 CX \\ Amsterdam, The Netherlands; ${ }^{2}$ Department of Clinical Oncology, Leiden University Medical Center, Albinusdreef 2, 2333 ZA Leiden, \\ The Netherlands
}

\begin{abstract}
Over the past decades, much has been learnt about the genes that contribute to oncogenic transformation of primary cells in vitro. However, much less is known about the genes that contribute to the later stages of tumor progression, in which cells of ever increasing malignancy arise through clonal selection in vivo. To search for genes that confer a tumor progression phenotype in vivo, we have used a functional genetic approach. We used adenovirus-transformed mouse embryo fibroblasts, which are tumorigenic in immunodeficient nude mice, but not in immunocompetent mice, due to strong cytotoxic T-cellmediated immune rejection. We infected these cells in vitro with several high-complexity retroviral cDNA expression libraries and selected rare variants that formed tumors in immunocompetent mice. Using this approach, we identify here the TRK-T3 oncogene as a tumor progression gene. TRK-T3 does not inhibit T-cell reactivity towards the tumor cells. Instead, we find that cells expressing TRK-T3 enhances in vivo growth rate, most likely by stimulating anchorage-independent proliferation in growth factor-limiting conditions. Our data indicate that cDNA expression libraries can be used to identify tumor progression genes in vivo that cannot be readily identified using in vitro cell culture systems.

Oncogene (2004) 23, 4959-4965. doi:10.1038/sj.onc.1207667 Published online 12 April 2004
\end{abstract}

Keywords: functional screen; tumor progression; cancer; transformation

\section{Introduction}

Oncogenic transformation of primary rodent cells in culture requires, in general, at least two collaborating oncogenes. In addition to these initial immortalizing and transforming events, cancer cells undergo a continuous clonal selection in vivo, in which ever more malignant clones evolve in a Darwinian selection. Tumor progres-

\footnotetext{
*Correspondence: R Bernards; E-mail: r.bernards@nki.nl

${ }^{3}$ Current address: Department Medical Oncology, Free University Medical Center, Amsterdam, The Netherlands

Received 17 December 2003; revised 16 February 2004; accepted 20 February 2004; published online 12 April 2004
}

sion involves a number of steps such as loss of contact inhibition, the ability to invade tissues, angiogenesis and acquisition of metastatic potential (Hanahan and Weinberg, 2000).

Relatively few in vivo models have been developed to identify genes involved in the later stage of cancer development. Slow-transforming retroviruses (lacking viral oncogenes) have been employed in the past to identify cellular proto-oncogenes by insertional mutagenesis in vivo (for a review, see Jonkers and Berns, 1996). Combining proviral tagging in $\mathrm{E} \mu$-Pim 1 or H2-Kmyc transgenic mice with transplantation of primary lymphomas has allowed the identification of frat- 1 as a gene involved in the progression of $\mathrm{M}-\mathrm{MuLV}$-induced T-cell lymphomas (Jonkers et al., 1997). A problem with such models is that it is not clear at which level these genes act in tumor progression. Recent work to circumnavigate these limitations has taken advantage of new technology, through which the activity of oncogenes or tumor-suppressor genes can be controlled (Chin et al., 1999; Pelengaris et al., 1999, 2002; Jonkers et al., 2001).

In this study, we have searched for tumor progression genes using retroviral cDNA expression libraries. Such 'gain-of-function' genetic screens are powerful tools to place genes functionally into cancer-relevant pathways (see Brummelkamp and Bernards, 2003 for a review). To date, this type of genetic screen has been employed mostly to identify genes using in vitro cell culture systems. However, identification of tumor progression genes is most readily performed in an in vivo model, in which the tumor cells only have to overcome one welldefined obstacle.

To identify genes involved in tumor progression, we used cells transformed by adenovirus. We have previously described the generation of cell lines transformed by chimeric plasmids that contain the E1A oncogene from the nononcogenic adenovirus type 5 and the E1B oncogene from the highly oncogenic adenovirus type 12 (Bernards et al., 1982). These cells have unusual tumorigenic properties in that they are very tumorigenic in immunodeficient nude mice, but not in immunocompetent syngenic mice. This lack of tumorigenicity in immunocompetent mice is explained by the finding that these cells are efficiently recognized and killed by cytotoxic $\mathrm{T}$ lymphocytes (CTL), which prevents their 
tumorigenicity in the presence of a fully functional immune system (Bernards et al., 1983; Schrier et al., 1983). The major epitopes of adenovirus early region 1 (E1)-transformed cells that are recognized by CTL are encoded by the E1A and E1B oncogenes (Kast et al., 1989; Toes et al., 1996). Therefore, escape from CTL killing by loss of the CTL epitope does not readily occur, as the E1 oncogenes cannot be downregulated by the tumor cell, as they act as the transforming agent in the tumor cell. This contributes to the consistent lack of tumorigenicity of Ad5 E1A-transformed cells in immunocompetent animals. Such a low spontaneous incidence of tumor formation makes these cells wellsuited to perform an in vivo genetic screen for tumor progression genes.

The $T R K-T 3$ oncogene was initially identified from a human papillary thyroid tumor and results from the fusion of the tyrosine kinase (TK) domain of the nerve growth factor receptor $(N T R K 1$, also known as $T R K A)$ on chromosome 1 to sequences of a novel gene designated TRK-fused gene (TFG) on chromosome 3 (Greco et al., 1995). The $68 \mathrm{kDa}$ TRK-T3 fusion oncoprotein displays a constitutive tyrosine kinase activity resulting in its capability to transform mouse NIH 3T3 cells (Greco et al., 1995, 1998). We describe here an unexpected and new role for TRK-T3 in tumor progression.

\section{Results}

A cell system to identify tumor progression genes in vivo

We have reported previously that rodent cells transformed by a chimeric adenovirus $5 \mathrm{E} 1 \mathrm{~A} /$ adenovirus 12 E1B plasmid have unique oncogenic properties. Such cells are highly oncogenic in immunodeficient nude mice, but fail to form tumors in immunocompetent rodents, due to strong cytotoxic $\mathrm{T}$ cell (CTL) reactivity against the E1A oncogene (Bernards et al., 1983; Schrier et al., 1983; Kast et al., 1989). It has been shown previously that tumor cells and viruses employ multiple strategies to bypass immune surveillance, including downregulation of the $\mathrm{MHC}$ class I antigens and inhibition of antigen processing by the proteasome (Bernards et al., 1986; Wiertz et al., 1996). We were therefore interested to use this adenovirus-based cell system to search for additional cancer-derived cDNAs that are involved in tumor progression by allowing bypass of CTL-dependent tumor cell killing in vivo. Bypass of CTL-mediated cell killing in vivo by an exogenously expressed gene can be caused in several distinct ways. First, the gene could suppress the reactivity of the CTL against the tumor cells. Alternatively, the gene could bring about a significant increase in the in vivo growth rate of the tumor cells, allowing the tumor to out-compete killing by the immune system.

To search for tumor progression genes, we first transformed primary mouse embryo fibroblasts (MEFs) of Balb/c and FVB genotype with the Ad5/12 hybrid
Table 1 Tumorigenicity assay

\begin{tabular}{lcc}
\hline Host & Genotype/no. of cells injected & Tumor incidence \\
\hline Athymic nude & Balb $/ \mathrm{c} / 2 \times 10^{6}$ & $6 / 6$ \\
Athymic nude & $\mathrm{FVB} / 2 \times 10^{6}$ & $6 / 6$ \\
Normal Balb/c & $\mathrm{Balb} / \mathrm{c} / 2 \times 10^{6}$ & $0 / 6$ \\
Normal FVB & $\mathrm{FVB} / 2 \times 10^{6}$ & $0 / 6$ \\
\hline
\end{tabular}

In all, $1.5 \times 10^{6} \mathrm{Balb} / \mathrm{c}$ or FVB Ad 5/12-transformed MEFs were injected subcutaneously into mice, as indicated. Tumors developed within 4 weeks in nude mice. Normal mice remained free of tumors for 4 months

early region 1 plasmid. Table 1 shows that both Ad5/12transformed Balb/c and FVB MEFs are tumorigenic in athymic nude mice with short latency period. As expected, injection of these cells in syngenic immunocompetent Balb/c or FVB mice did not result in tumor formation (Table 1). This lack of tumorigenicity allowed for the screening of genes that enable the Ad5/12MEFs to form tumors in immunocompetent mice.

\section{A functional genetic screen for tumor progression genes}

To search for genes that allow Ad5/12-transformed MEFs to form tumors in immunocompetent mice, we used a functional genetic screen. We infected Ad5/12tranformed MEFs of Balb/c genotype in vitro with two high-complexity retroviral cDNA expression libraries (Shvarts et al., 2002). After a limited expansion of the infected cells in vitro, cells were trypsinized, washed twice in PBS and injected subcutaneously into immunocompetent syngeneic mice. In all, $4 \times 10^{6}$ of cDNA library-infected Ad5/12-transformed MEFs were injected per mouse flank and followed for tumor development.

Table 2 shows that after cDNA library infection, we obtained two independent tumors from cells infected with one fraction ( $>3 \mathrm{~kb}$ cDNAs) of the human K562 erythroleukemia cDNA library and one tumor from the JEG human choriocarcinoma ( $>3 \mathrm{~kb}$ cDNA fraction) cDNA library (see Table 2). One tumor originating from mock-infected cells was detected. This might be due to the fact that Ad512 cells are functionally p53 deficient, causing genomic instability and thus allowing spontaneous tumor-promoting mutations to accumulate at low frequency. To investigate which cDNAs might be responsible for the tumorigenic properties of the retrovirally transduced cells, we recovered the retroviral cDNA inserts from genomic DNA isolated from two of the mouse tumors using PCR with primers specific for the retroviral vector. Figure 1 shows that tumor M2 has two distinct cDNA inserts of 2.7 and $1.5 \mathrm{~kb}$ in size and was therefore investigated further. These cDNA inserts were recloned into the retroviral vector and their identity was revealed by DNA sequence analysis. This indicated that the $2.7 \mathrm{~kb}$ cDNA encoded a full-length copy of the TRK-T3 oncogene, whereas the $1.5 \mathrm{~kb}$ cDNA species encoded a truncated form of CDK4 (basepair 228-1139), referred to below as $\triangle \mathrm{CDK} 4$. 
Table 2 In vivo functional genetic screen

\begin{tabular}{lcccrr}
\hline$c D N A$ library infected & Mock & K562 l-3kb & K562 >3kb & $J E G 1-3 k b$ & $J E G>3 k b$ \\
\hline Tumorigenicity in Balb/c mice & $1 / 24(4 \%)$ & $0 / 24(0 \%)$ & $2 / 12(16.1 \%)$ & $0 / 12(0 \%)$ & $1 / 12(8.3 \%)$ \\
\hline
\end{tabular}

Ad512-transformed Balb/c MEFs were infected with the indicated cDNA expression libraries and infected cells were injected into immunocompetent Balb/c mice. Tumor incidence was monitored

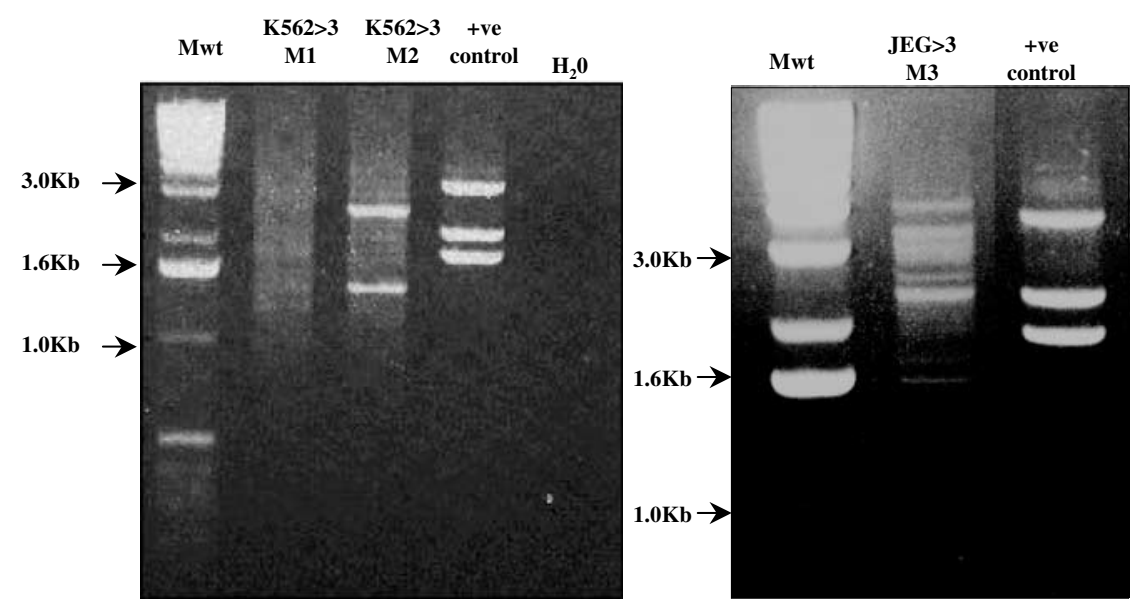

Figure 1 Recovery of retrovirally transduced cDNAs. Proviral cDNA inserts were recovered from genomic DNA of the tumors using specific primers for the retroviral vectors, and separated by agarose gel electrophoresis. Tumor M2 contains two clear cDNA inserts at 2.7 and $1.5 \mathrm{~kb}$ in size (left panel, lane 3). These inserts were recloned into retroviral vector of the library and identified as TRK-T3 and a truncated CDK4 (228-1139 bp), termed $\triangle \mathrm{CDK} 4$, respectively. Lane marked + ve control: a positive control sample containing retroviral vectors with three known inserts. Lane labeled $\mathrm{H}_{2} \mathrm{O}$ : negative control PCR reaction

\section{TRK-T3 expression enhances tumorigenicity}

In principle, both TRK-T3 and $\triangle \mathrm{CDK} 4$ could contribute to enhanced tumorigenesis. For instance, it has been reported that TRK-T3 has transforming activity on NIH 3T3 cells (Roccato et al., 2002), whereas elevated expression of CDK4 collaborates with an activated $R A S$ oncogene in epidermal tumorigenesis (Lazarov et al., 2002). To investigate as to which of the two cDNAs present in tumor M2 was responsible for the induction of tumorigenic capacity in immunocompetent mice, we expressed TRK-T3 and $\Delta$ CDK4 separately in Ad5/12-transformed Balb/c MEFs by retroviral transduction. Western blot analysis of infected Ad5/12 MEFs revealed the presence of TRK-T3 and of a truncated $24 \mathrm{kDa}$ CDK4 species, which fits the predicted size of the $\triangle \mathrm{CDK} 4 \mathrm{cDNA}$ from the cDNA library (Figure 2a). Immunohistochemical staining of Ad5/12 MEFs infected with TRK-T3 demonstrates TRK-T3 expression in the membrane and cytoplasm of cells (Figure 2b).

The TRK-T3- and $\Delta$ CDK4-expressing Ad5/12 MEFs were then injected into immunocompetent Balb/c mice. Table 3 shows the rather unusual kinetics of tumor development that was observed. In the first instance, some $30-40 \%$ of cells expressing $T R K-T 3$ or coexpressing $T R K-T 3$ and $\triangle C D K 4$ induced clearly palpable tumors of $3-5 \mathrm{~mm}$ diameter in size in the first 10 days following injection. In contrast, only $7 \%$ of the mockinfected cells and $19 \%$ of the $\triangle C D K 4$-expressing cells yielded initial tumors. However, many of these tumors began to shrink after approximately 10 days and were completely regressed after $2-3$ weeks. The timing of the tumor regression is consistent with the time required by the host to mount a specific CTL response against the adenoviral epitopes expressed on the tumor cells. This suggests that tumor regression might be caused by CTLmediated rejection by the host's immune system. Nevertheless, some $14 \%$ of the tumors (TRK-T3-expressing cells) or $12.5 \%$ of the tumors (TRK-T3 $+\Delta \mathrm{CDK} 4-$ expressing cells) grew progressively to more than $2 \mathrm{~cm}$ diameter within 4-5 weeks, after which the animals were euthanized. In contrast, only some $5 \%$ of the mock- or $\Delta \mathrm{CDK} 4$-infected cells gave rise to progressively growing tumors. These data suggest that expression of the $T R K$ $T 3$ oncogene enhances the tumorigenicity of $\mathrm{Ad} 5 / 12-$ transformed cells in immunocompetent mice. The penetration of the phenotype was only partial as not all animals injected with the TRK-T3-expressing cells formed tumors.

\section{TRK-T3 and CDK4 do not modulate sensitivity to the immune system}

The observation that many tumors induced by cells expressing $T R K-T 3$ regressed with kinetics that are consistent with CTL rejection of the tumors suggests that expression of TRK-T3 does not suppress CTL reactivity against the adenoviral epitopes. To test this 
4962

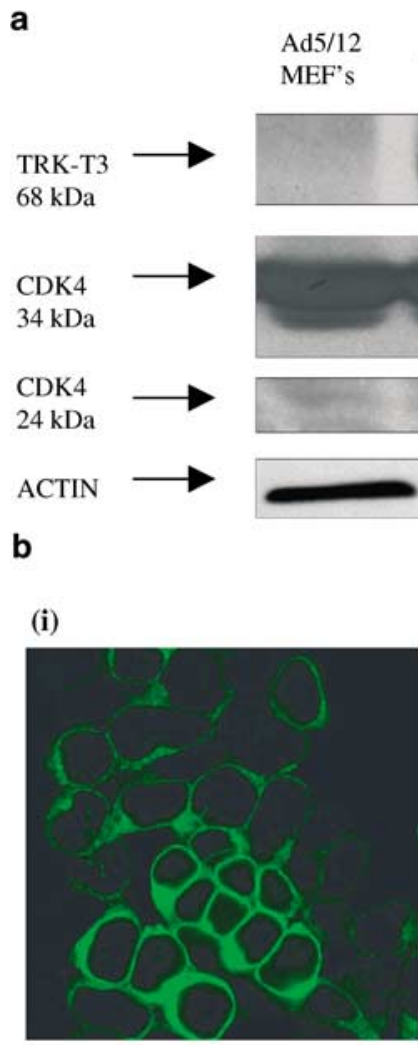

(ii)
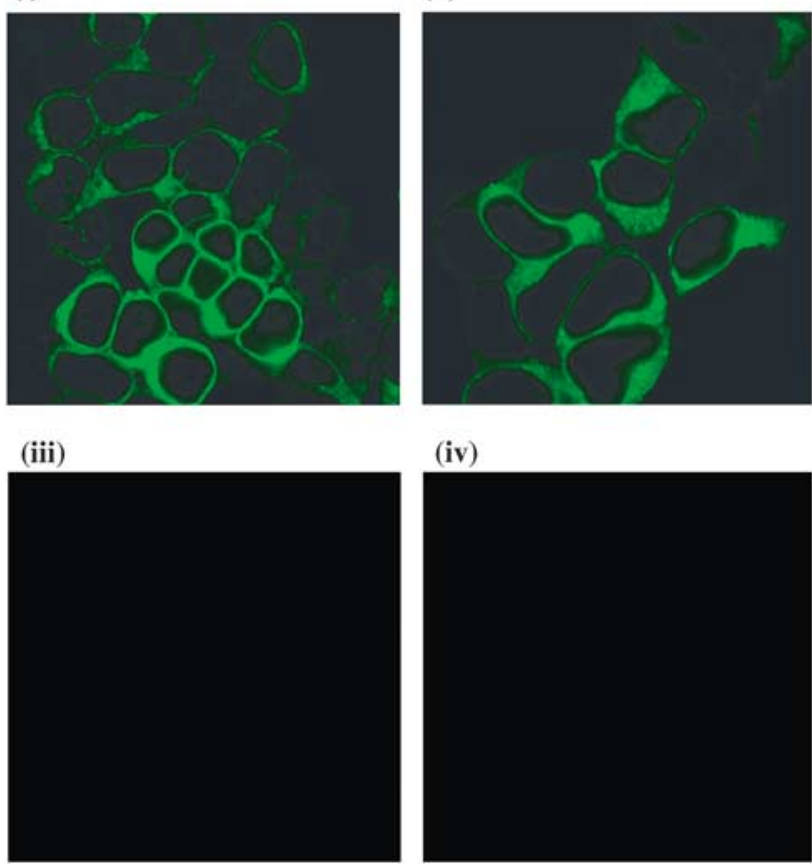

(iv)

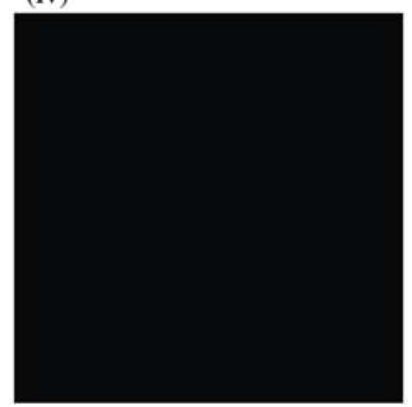

Figure 2 Expression of retrovirally transduced oncogenes. (a) Western blot analysis of infected Ad5/12 MEFs with TRK-T3 and/ or $\triangle \mathrm{CDK} 4$ demonstrating overexpression of TRK-T3 and CDK4. Shown are Western blots of total cell lysates transduced with the indicated retroviral vectors (above the lanes) and probed with antibodies against TRK-T3 or CDK4. Actin antibody served as a loading control. Overexpression of a $24 \mathrm{kDa}$ CDK4 species was detected that fits the predicted size of the truncated form of the cDNA isolated from the cDNA library. $\triangle \mathrm{CDK} 4$ : truncated cDNA CDK4 from cDNA library screen. (b) Cellular localization TRKT3 protein in Ad5/12 MEFs transduced with TRK-T3. Panels (i) and (ii): TRK-T3 expression in the membrane and cytoplasm of cells at $\times 630$ and $\times 1260$ magnification, respectively. Panel (iii): no primary antibody control and panel (iv): Ad 5/12 MEFs not infected with TRK-T3 oncogene

directly, we measured the ability of Ad5 E1A-specific CTL to lyse Ad5/12 MEFs expressing the TRK-T3 oncogene in vitro (Figure 3a). We observed that cells expressing either $T R K-T 3$ or $\triangle C D K 4$ or both were as sensitive to CTL lysis in vitro as parental Ad5/12 MEFs or mock-infected Ad5/12 MEFs. These results argue against the model that expression of $T R K-T 3$ suppressed
CTL reactivity against the tumor cells. However, we cannot exclude that $T R K-T 3$ expression allows tumor cells to evade CTL in vivo in a way that cannot be readily measured in vitro.

\section{TRK-T3 confers proliferative advantage in vivo}

An alternative explanation for the observed increase in tumorigenicity by $T R K-T 3$ is that expression of this oncogene enhances the in vivo proliferation rate of $\mathrm{Ad} 5$ 12-transformed MEFs, thereby enabling the tumor cells to out-compete the immune system. To address this, we tested the ability of $T R K-T 3 \mathrm{Ad} 5 / 12 \mathrm{MEF}$ to grow in athymic nude Balb/c mice. Figure $3 b$ shows that expression of $T R K-T 3$ causes rapid tumor formation in vivo within 2 weeks of injection. Coexpression of $T R K$ $T 3$ and $\triangle C D K 4$ did not lead to a further increase in tumorigenicity, consistent with the results shown above. Postmortem analysis of the tumors revealed that $T R K$ $T 3$ tumors were very vascular (data not shown). The significance of this is at present not clear. These data are consistent with the model that expression of $T R K-T 3$ enhances in vivo growth rate, conferring a selective advantage onto $T R K$ - T3-expressing cells.

\section{TRK-T3 confers proliferative advantage in vitro}

Given that $T R K-T 3$ caused tumor progression in vivo and did not modulate a CTL cell response, we tested whether the TRK-T3 oncogene conferred an increased proliferation rate in vitro under conditions that resemble those that are encountered by the tumor cells after injection at a subcutaneous site (i.e. mitogen-deprived and anchorage-independent growth conditions). Figure 4a shows that Ad5/12-transformed MEFs expressing $T R K-T 3$, but not parental cells of mockinfected cells, were able to form colonies in medium containing $0.5 \%$ serum. The colonies formed large cellular columns in the center (see Figure 4a, right panels), which grew out into the media and broke off if the plate was shaken. When these broken off colonies were transferred to new plates, they reformed new colonies, indicating that the cell mass in the center of the transformed colony is viable (data not shown). This suggests that the cells have lost their anchorage dependence. To test this directly, we measured the ability of TRK-T3-expressing Ad5/12-transformed MEFs to grow in semisolid media in low serum conditions. Figure $4 \mathrm{~b}$ shows that $T R K-T 3$ causes anchorage-independent growth in soft agar assays in low serum conditions, which was not observed in parental or mock-infected Ad5/12 MEFs. We conclude that $T R K-T 3$ acts as a tumor progression gene by enhancing proliferation of tumor cells under mitogendeprived and anchorage-independent conditions.

\section{Discussion}

We describe here the design and use of an in vivo 'gainof-function' genetic screen to identify genes that act in 
Table 3 Tumor incidence with identified oncogenes

\begin{tabular}{lcccc}
\hline Balb/c mice, 3-4 weeks & PMX-GFP or no treatment & $\Delta C D K 4$ & TRK-T3 & TRK-T3 and $\Delta C D K 4$ \\
\hline Incidence of tumor take & $5 / 71$ & $4 / 21$ & $7 / 21$ & $10 / 24$ \\
(\%) & 7 & 19 & 33.3 & 41.6 \\
No. of tumors regressed & $1 / 71$ & $3 / 21$ & $4 / 21$ & $7 / 24$ \\
No. of tumors that grew progressively & $4 / 71$ & $1 / 21$ & $3 / 21$ & $3 / 24$ \\
$(\%)$ & 5.6 & 4.8 & 14.3 & 12.5 \\
\hline
\end{tabular}

In all, $4 \times 10^{6}$ cells expressing the indicated oncogenes were subcutaneously injected in the flanks of immunocompetent Balb/c mice. $\Delta \mathrm{CDK} 4$ : truncated cDNA from cDNA library screen

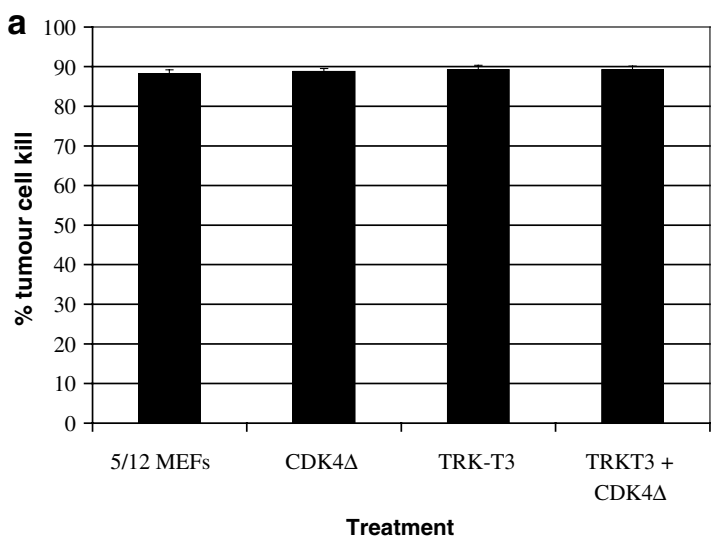

b

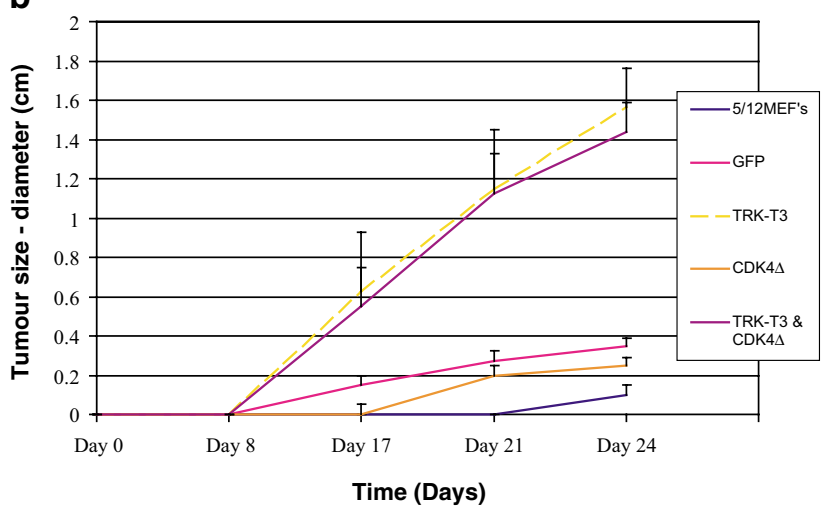

Figure 3 Characterization of TRK-T3-expressing cells. (a) Ad5/12 MEFs overexpressing TRK-T3 and/or $\triangle$ CDK4 were incubated with Ad5-E1A-specific CTL and tumor cell killing was measured. (b) Tumorigenicity assay in athymic mice. Cells expressing the indicated oncogenes were injected subcutaneously into nude mice and tumor development was measured over time. In all, $5 \times 10^{5}$ cells were injected with or without TRK-T3 or $\triangle \mathrm{CDK} 4$ to discriminate growth rates of untreated Ad5/12 MEFs to those cells with genes of interest. $\triangle \mathrm{CDK} 4$-infected Ad5/12 MEFs grew only as fast as controls, whereas TRK-T3 or TRK-T3 plus $\Delta$ CDK 4 grew very rapidly to $1 \mathrm{~cm}$ within 2 weeks of subcutaneous injection

the later stages of tumor progression. We used cells that are fully transformed by most criteria (i.e. immortality in vitro and tumorigenic in nude mice), but failed to form tumors in immunocompetent hosts, due to rejection by CTL. Introduction of retroviral cDNA expression libraries in such cells, followed by in vivo selection allowed the identification of the TRK-T3 oncogene as a tumor progression gene that confers an oncogenic phenotype in a host having a fully functional immune system. TRK-T3 is commonly expressed in human papillary thyroid carcinoma and has transforming activity in NIH 3T3 cells (Greco et al., 1995).

We envisaged that the in vivo screen might either identify genes that enable tumor cells to escape from the host immune surveillance or identify genes that confer a significant proliferative advantage in vivo, such that the tumor cells can out-compete the immune system of the host. Several lines of evidence suggest that TRK-T3 does not interfere with the recognition of the tumor cells by the host immune system. First, the ability of tumor cell-specific CTL to lyse tumor cells was not affected by expression of TRK-T3 (Figure 3a). Furthermore, expression of MHC class I antigens on tumor cells was unaltered by expression of TRK-T3 (data not shown). Finally, the in vivo kinetics of tumor regression of $T R K$ T3 expressing cells are also consistent with a fully functional immune response against the tumor cells. Instead, the data presented here are consistent with the notion that expression of $T R K-T 3$ confers a dramatic in vivo proliferative advantage onto the tumor cells, which enables the tumor cells to overcome immune rejection. For instance, we observed that expression of $T R K-T 3$ significantly enhanced proliferation rates of tumor cells in immunodeficient nude mice (Figure $3 b$ ). This is most likely due to the fact that TRK-T3-expressing cells grow in semisolid media and in growth-factor poor medium (Figure 4). The ability of the TRK-T3 oncogene to stimulate cell proliferation in a ligand-independent manner is well-documented. The TFG portion of TRK-T3 contains a coiled-coil domain most likely responsible for the constitutive, ligand-independent activation of the receptor tyrosine kinase activity (Roccato et al., 2002). Further mapping of the TFG sequence of TRK-T3 revealed binding motifs for PKC, CK2 and SH3 (Mencinger et al., 1997), which could activate cell survival pathways. It has been demonstrated that TRK-T3 can activate various signal transduction pathways in transformed cells including PLC, JNK and ERK1/2 MAP kinases (Roccato et al., 2003). Activation of such signaling pathways may well explain why expression of $T R K-T 3$ allows tumor cells to proliferate in growth factor-deprived conditions.

However, the proliferative advantage conferred by expression of $T R K-T 3$ does not always decide the battle between the host immune system and the tumor in favor of the tumor, as regression of established tumors was frequently observed in immunocompetent mice, but never in immunodeficient nude mice (Table 3, 
a i

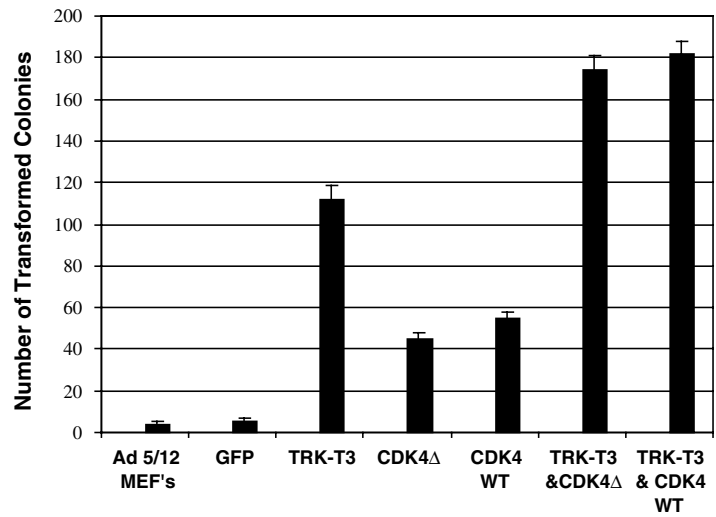

b

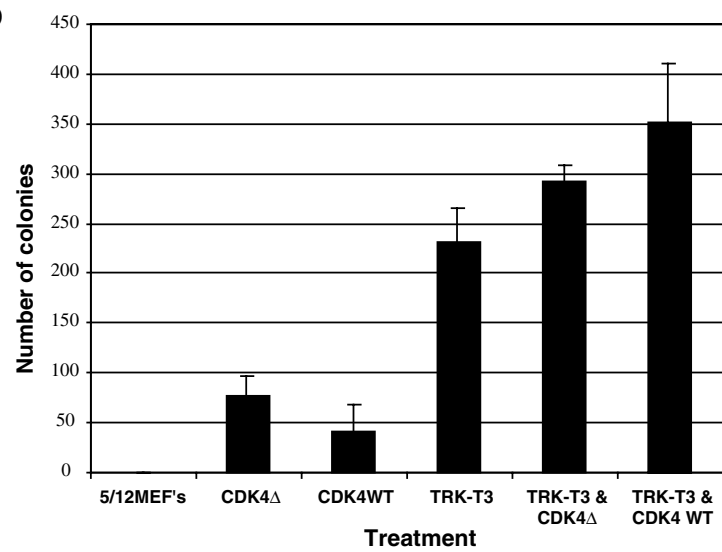

ii

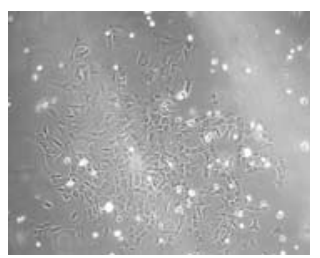

iii

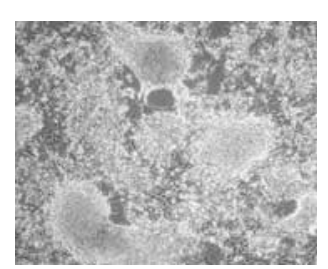

Figure 4 In vitro growth properties of TRK-T3 expressing cells. (a) Low serum in vitro colony formation assay. Panel (i): cells expressing the indicated oncogenes were seeded in media containing $0.5 \%$ serum. The number of colonies was counted after 4 weeks. Panel (ii): photograph of parental Ad 5/12 MEFs maintained for 4 weeks in low serum (Magnification $\times 200$ ). Panel (iii): photograph of TRK-T3 MEFs after 4 weeks in low serum (Magnification $\times 200)$. (b) In vitro soft agar assay in low serum $(1.0 \%)$. Cells expressing the indicated oncogenes were plated in soft agar in low serum conditions. The number of colonies that were visible after 2 weeks is graphically represented

Figure 3b). Interestingly, spontaneous tumor regression is also seen in the clinic, especially for tumors that induce tumor-specific CTL responses, such as melanoma (Coulie and van der Bruggen, 2003). Interestingly, neurotrophin receptors of the TRK family are expressed in primary human melanoma (Xu et al., 2003), raising the possibility that expression of these genes allows the tumor cells to overcome the CTL response provoked by the melanoma tumor antigens.

\section{Materials and methods}

\section{Cell culture, transfection, infection}

All cells were cultured in Dulbecco's modified Eagle's medium supplemented with $8 \%$ fetal calf serum (FCS). Low serum cultures were performed at $0.5 \%$ FCS. Ecotropic retroviral supernatants were produced by transfection of Phoenix packaging cells by calcium phosphate precipitation. At $48 \mathrm{~h}$ post-transfection, the tissue culture medium was filtered through a $0.45 \mu \mathrm{m}$ filter, and the viral supernatant was used. In all, $1 \times 10^{6} \mathrm{Ad} 5 / 12 \mathrm{Balb} / \mathrm{c}$ mouse embryo fibroblasts (MEFs) were infected with the human K562 erythroleukemia or JEG choriocarcinoma cDNA libraries (Shvarts et al., 2002) in two rounds of infections in media supplemented with $4 \mu \mathrm{g}$ / $\mathrm{ml}$ polybrene. Cells were washed the following day and expanded. For tumorigenicity assays, cells were trypsinized, washed twice in PBS and injected subcutaneously. In total, $4 \times 10^{6}$ cells infected with either the K562 or JEG cDNA libraries were injected per mouse flank.

\section{Creation of Ad5/12-transformed cells}

To create an Ad5/12-transformed cell line, primary MEFs were isolated from embryos of both FVB and Balb/c genetic background and transfected with a chimeric plasmid harboring the early region $1 \mathrm{~A}$ of adenovirus 5 (Ad5 E1A) and early region $1 \mathrm{~B}$ of adenovirus 12 (Ad12 E1B), the construction of which has been described previously (Bernards et al., 1982).

\section{Recovery of retrovirally encoded $c D N A s$}

Tumor was excised after growing to $1 \mathrm{~cm}$ in diameter, cut with sterile scissors and trypsinized for $20 \mathrm{~min}$ at $37^{\circ} \mathrm{C}$. The solution was allowed to settle for $10 \mathrm{~min}$ and the cell suspension returned to a $10 \mathrm{~cm}$ cell culture plate. A sample of the cell suspension was taken for genomic DNA isolation using DNAzol. Expand PCR was performed on genomic DNA from the tumor using specific primers for the PMX vector. 


\section{Western blot, immunohistochemistry}

Western blots were carried out on whole-cell extracts, separated on 10-12\% SDS-polyacrylamide gels and transferred to polyvinylidene difluoride membranes (Millipore). Western blots were developed using enhanced chemiluminescence (Amersham Biosciences, Inc.) following the instructions of the manufacturer. The TRK antibody was obtained from Research Diagnostics Inc. (USA).

For immunofluorescence, cells were fixed in $4 \%$ paraformaldehyde and stained. Second antibodies used was fluorescein isothiocyanate-conjugated goat anti-mouse used at $1: 75$ dilution (DAKO). Images were visualized using the confocal microscope facility at the NKI.

\section{In vitro CTL lysis assay}

Ad5/12-transformed MEFs were infected with TRK-T3, $\triangle \mathrm{CDK} 4$, coinfected with TRK-T3 and $\triangle \mathrm{CDK} 4$, or with GFP-encoding retrovirus (control). CTLs that specifically recognize the E1A antigen were added at an effector to target ratio (E: T ratio) of $1: 1$ in six-well plates. Cells were incubated overnight and the next day washed with PBS and cell survival assessed by counting the number of viable tumor cells.

\section{Low serum colony formation assay}

Colony formation in low serum was performed by plating $5 \times 10^{5}$ cells in a $10 \mathrm{~cm}$ plate. The next day, cells were washed and media with $0.5 \%$ serum placed on cells for 4 weeks. After this, plates were washed and fixed in 3:1 methanol/acetic acid for $10 \mathrm{~min}$ and stained with $0.5 \%$ Coomassie blue for $30 \mathrm{~min}$, washed in PBS and air-dried $\mathrm{O} / \mathrm{N}$. The number of transformed colonies was counted in 10 fields at objective $\times 10$.

\section{Soft agar assay}

In all, $2 \times 10^{4}$ cells were resuspended in $2 \mathrm{ml} \mathrm{0.4 \%} \mathrm{low-melting-}$ point agarose (Sigma type VII, Catalog No. A-4018) and seeded, in duplicate, into six-well plates coated with $1 \%$ lowmelting-point agarose in DMEM containing either 10 or $1 \%$ serum. The number of foci was scored after 2 week's culture at $37^{\circ} \mathrm{C}$.

\section{Tumorigenicity assay}

Ad5/12-transformed MEFs were infected with TRK-T3, $\triangle \mathrm{CDK} 4$, both TRK-T3 and $\triangle \mathrm{CDK} 4$ together, or GFP (control). In total, $5 \times 10^{5}$ cells were injected subcutaneously per nude mouse flank and tumor size was measured over 4 weeks. A low number of cells was injected to discriminate between the normal transformed growth of Ad5/12 Balb/c MEFs and increased rate of growth with TRK-T3- or CDK4infected cells.

\section{Acknowledgements}

We thank Roderik Kortlever and Thijn Brummelkamp for technical advice. The animal work was assisted by animal technicians Louis Tolkamp and Henk Starrevelt from the animal facility at the NKI. This work was supported by a grant from the Dutch Cancer Society (KWF) and the Netherlands Organization for Scientific Research (NWO).

\section{References}

Bernards R, Dessain SK and Weinberg RA. (1986). Cell, 47, 667-674.

Bernards R, Houweling A, Schrier PI, Bos JL and van der Eb AJ. (1982). Virology, 120, 422-432.

Bernards R, Schrier PI, Houweling A, Bos JL and van der Eb AJ. (1983). Nature, 305, 776-779.

Brummelkamp TR and Bernards R. (2003). Nat. Rev. Cancer, 3, 781-789.

Chin L, Tam A, Pomerantz J, Wong M, Holash J, Bardeesy N, Shen Q, O'Hagan R, Pantginis J, Zhou H, Horner II JW, Cordon-Cardo C, Yancopoulos GD and DePinho RA. (1999). Nature, 400, 468-472.

Coulie PG and van der Bruggen P. (2003). Curr. Opin. Immunol., 15, 131-137.

Greco A, Fusetti L, Miranda C, Villa R, Zanotti S, Pagliardini S and Pierotti MA. (1998). Oncogene, 16, 809-816.

Greco A, Mariani C, Miranda C, Lupas A, Pagliardini S, Pomati M and Pierotti MA. (1995). Mol. Cell. Biol., 15, 6118-6127.

Hanahan D and Weinberg RA. (2000). Cell, 100, 57-70.

Jonkers J and Berns A. (1996). Biochim. Biophys. Acta., 1287, $29-57$.

Jonkers J, Korswagen HC, Acton D, Breuer M, Berns A and Scheijen B. (1997). EMBO J., 16, 441-450.

Jonkers J, Meuwissen R, van der Gulden $\mathrm{H}$, Peterse $\mathrm{H}$, van der Valk M and Berns A. (2001). Nat. Genet., 29, 418-425.

Kast WM, Offringa R, Peters PJ, Voordouw AC, Meloen RH, van der Eb AJ and Melief CJ. (1989). Cell, 59, 603-614.

Lazarov M, Kubo Y, Cai T, Dajee M, Tarutani M, Lin Q, Fang M, Tao S, Green CL and Khavari PA. (2002). Nat. Med., 8, 1105-1114.

Mencinger M, Panagopoulos I, Andreasson P, Lassen C, Mitelman F and Aman P. (1997). Genomics, 41, 327-331.

Pelengaris S, Khan M, Evan G and Evan GI. (2002). Nat. Rev. Cancer, 2, 764-776.

Pelengaris S, Littlewood T, Khan M, Elia G and Evan G. (1999). Mol. Cell, 3, 565-577.

Roccato E, Miranda C, Ranzi V, Gishizki M, Pierotti MA and Greco A. (2002). Br. J. Cancer, 87, 645-653.

Roccato E, Pagliardini S, Cleris L, Canevari S, Formelli F, Pierotti MA and Greco A. (2003). Oncogene, 22, 807-818.

Schrier PI, Bernards R, Vaessen RT, Houweling A and van der Eb AJ. (1983). Nature, 305, 771-775.

Shvarts A, Brummelkamp TR, Scheeren F, Koh EY, Daley GQ, Spits H and Bernards R. (2002). Genes Dev., 16, 681-686.

Toes RE, Blom RJ, van der Voort E, Offringa R, Melief CJ and Kast WM. (1996). Cancer Res., 56, 3782-3787.

Wiertz EJHJ, Jones TR, Sun L, Bogyo M, Geuze HJ and Ploegh HL. (1996). Cell, 84, 769-779.

Xu X, Tahan SR, Pasha TL, Zhang PJ, Marchetti D, Murry B, Galjour J, Wilke-Greiter A, Fanburg-Smith JC, Miettinen M, Innominato PF, Libbrecht L, van den Oord JJ, Kramer K, Gerald W, LeSauteur L, Saragovi HU, Cheung NK, Lafarge-Frayssinet C, Daya-Grosjean L, Estrade S, Frezouls G, Sarasin A, Cassingena R, Easty DJ, Herlyn M and Bennett DC. (2003). J. Cutan. Pathol., 30, 318-322. 